\title{
Wireless Technology and System Integration in Body Area Networks for m-Health Applications
}

\author{
Emil Jovanov \\ Electrical and Computer Engineering Department \\ University of Alabama in Huntsville, Huntsville, Alabama, U.S.A.
}

\begin{abstract}
Health integrates mobile computing, medical sensor, and communications technologies for mobile health-care applications. Wireless Body Area Networks (WBANs) of intelligent sensors represent an emerging technology for system integration with great potentials for unobtrusive ambulatory health monitoring during extended periods of time. However, system designers will have to resolve a number of issues, such as severe limitations of sensor weight and size necessary to improve user's compliance, sensor resource constraints, intermittent availability of uplink connectivity, reliability of transmission, security, and interoperability of different platforms.

We present current and emerging wireless technologies and developments in pervasive and mobile technologies that are vital for implementation of WBAN-based monitors and m-Health system integration. We emphasize the problem of reliable system operation with extremely low power consumption and discontinuous connectivity, which are typical for ambulatory monitoring.
\end{abstract}

Keywords-Wireless Technology, Body Area Networks, m-Health, Ambulatory Monitoring, Sensors

\section{INTRODUCTION}

The emerging m-Health concept represents the evolution of e-health systems loosely defined as the use of the Internet for healthcare to mobile applications without guaranteed Internet connectivity [1]. The advances in m-Health systems are driven by the developments in wireless communications, pervasive, and wearable technologies [2]. They share the same ultimate design goals: minimization of weight and size of sensors that are critical for user's acceptance, portability, unobtrusiveness, ubiquitous connectivity, reliability, and seamless system integration. Global connectivity is important for improved handling of emergency situation, but also for an increasingly important user segment informal caregivers. A 1997 study found that almost one third of US adults, most of whom held full-time jobs, were serving as informal caregivers - mostly to an elderly parent [3].

A wearable health-monitoring device using a Personal Area Network or Body Area Network can be integrated into a user's clothing. This system organization, however, is unsuitable for lengthy, continuous monitoring, particularly during normal activity, such as intensive training or computer-assisted rehabilitation. The most recent phase that provides patient mobility and unobstructed use came with the introduction of wireless, intelligent, and implantable sensors. A typical Wireless Body Area Network (WBAN) consists of a number of inexpensive, lightweight, and miniature sensor platforms, each featuring one or more physiological sensors, e.g. motion sensors, electrocardiograms (ECGs), electromyograms (EMGs), and electro-encephalograms (EEGs). Typical example of this type of application is ambulatory monitoring of user's activity [4][5]. The sensors could be located on the body as tiny intelligent patches, integrated into clothing, or implanted below the skin or muscles.

In this paper we present main issues and technical challenges of design and system integration of WBAN based m-Health systems.

\section{WIRELESS TECHNOLOGY}

In order to succeed on the market, wireless medical sensors will be required to have a very low price and high volume. Therefore, we can expect that the future solutions will be based on standard wireless technologies [6]. The most widely used currently commercially available WBAN technologies include Bluetooth [7] and ZigBee [8]. Bluetooth is mature technology, already integrated in many cell phones and Personal Digital Assistant (PDA) devices. It allows high communication bandwidth of up to $720 \mathrm{kbps}$, which is more than sufficient for most intelligent sensors. However, in spite of low-power modes, power consumption and complexity of protocol stack implementation is still a limiting factor for most WBAN applications.

ZigBee is an emerging wireless standard for low data rate, very low-power applications, with potential applications in home automation, industrial control, and personal health care. The maximum data rate of $250 \mathrm{kbps}$ is still sufficient for intelligent health monitoring sensors.

Other emerging wireless technologies, such as Ultra Wide Band and Wireless USB will likely influence wearable health monitoring applications. However, their goal is high bandwidth with reduced power consumption for personal area networks and home networks. Therefore, they are very good candidates for the personal server communication, rather than individual sensors in WBAN. Several research projects introduce wireless solutions much better suited for intelligent sensors. For example, MEMS resonators offer extremely low power consumption in the order of $100 \mu \mathrm{W}$ [9] that will be ideal for wireless sensors implemented as intelligent patches. 


\section{SySTEM INTEGRATION}

Success of wearable health monitoring systems crucially depends on system integration. Seamless integration of personal health monitoring systems will determine user's acceptance and compliance, while integration of measurements and events into electronic medical records and health medical systems can gain support of medical professionals.

Typical example of data flow in full hierarchy m-Health systems is represented in Fig. 1 (adapted from [5]). A set of sensors is integrated into WBAN and communicate with the personal server or directly to the Internet Gateway. All information is then stored in Electronic Medical Record (EMR) or emergency server through the Internet. Different configurations can exploit different parts of the price/power/performance design space.

The lowest level of hierarchy consists of intelligent physiological sensors integrated into a WBAN. Current state of technology allows intelligent sensor nodes with highly integrated microcontrollers (systems on a chip), allowing the following sensor platform characteristics:

- processing power - 1-10 MIPS (Millions of Instruction Per Second)

- working memory (RAM) - 1 - $10 \mathrm{~KB}$

- on chip flash memory (program and secondary data storage) - 10-100 KB

- integrated peripherals (Timers, Counters, PWM, A/D, $\mathrm{D} / \mathrm{A}$, and communication controllers).

- external flash memory storage with capacity in the order of $1 \mathrm{MB}$.

- very low power consumption $(1-10 \mathrm{~mW}$ for processing, less than $100 \mu \mathrm{W}$ in idle mode, and 20-50 $\mathrm{mW}$ during wireless communication)

Available memory and processing power of commercially available sensor platforms allows real-time signal processing and transmission of results and detected events for many health monitoring applications. Symbolic flow of data from sensors is represented as queues of messages in Fig. 1. A sensor platform first stores unacknowledged messages and events in local memory (RAM) and/or internal or external flash memory. This allows crucial power and reliability advantage of intelligent sensors over earlier generations of telemetric systems with transmission of raw signals.

All messages from sensors are collected by the network controller and processed on a personal server. In some applications sensors can talk directly to the Internet Gateway (e.g. home monitoring). A personal server application can run on a PDA, cell phone, or home personal computer. Network controller could be an add-on device or integrated into the personal server (for example Bluetooth enabled cell phones). The PS processes individual event messages from sensors, stores segments of raw signals generated on request, and creates a synergy of information from individual sensors. Typically, all received messages are saved and retransmitted to the medical server, with additional messages created on the personal server. This information creates a new queue of messages and records

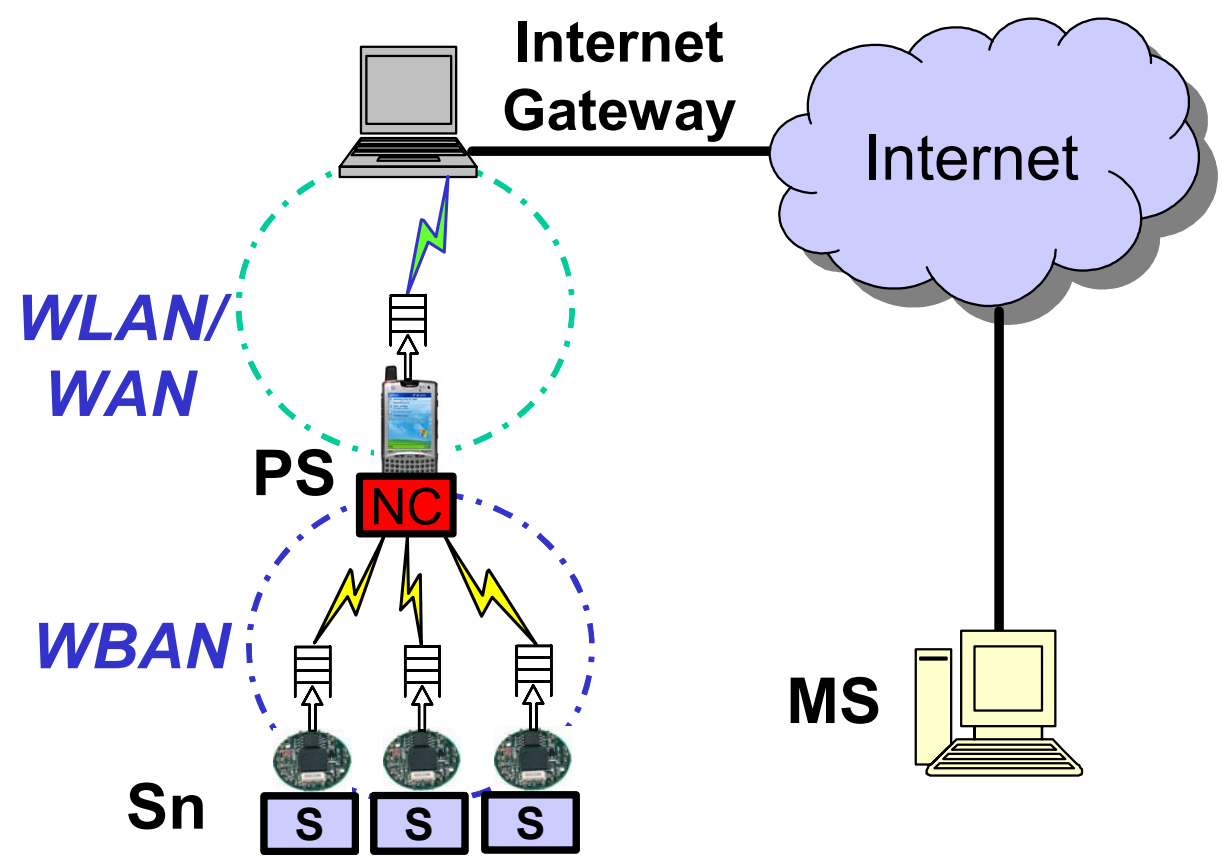

Fig. 1. WBAN system integration [5]. Legend: Sn - Physiological sensors, WBAN - Wireless Body Area Network, PS - Personal Server, WLAN - Wireless Local Area Network, WAN - Wide Area Network, MS - Medical Server, NC - WBAN network coordinator. 
coming from the personal server, as represented in Fig. 1. Performance characteristics of the personal server and the usually outperform individual sensors for at least one order of magnitude. Therefore, maintaining the queue of messages on the personal server for reliable transmission should not be a problem for most systems.

Communication between the personal server and the Internet Gateway as accomplished using standard WLAN and WAN technologies, such as 802.11 WLAN family, GPRS/GSM, UMTS, and other wireless local and wide area network technologies [1].

Final processing of messages and events is executed on the medical server(s). It is important to note that due to intermittent communication and message retransmissions in the m-Health hierarchy the medical server will have to process out of order messages. Therefore, it is necessary to precisely time stamp each event or segment of raw signal. All records must be organized to prevent disclosure of user's identity.

\section{Discussion AND CONCLUSIONS}

New generation of m-Health systems for continuous ambulatory monitoring raises a number of challenging issues that include:

- Need for extremely low power operation, low weight, and small size

- Non-invasive and unobtrusive operation

- Sensor flexibility necessary to adapt to the user's state and changes in the environment

- Seamless connectivity necessary for sensor integration into the monitoring system

- System awareness of environmental and patient factors associated with the use of wearable sensors in normal living conditions

- Secure and reliable communication and data storage

- Fault tolerant system operation, capable of adapting to faults of individual sensors and retransmission of lost packets
WBAN based m-Health technologies have great potential for continuous monitoring in ambulatory settings, early detection of abnormal conditions, and supervised rehabilitation. They can provide patients with increased confidence and a better quality of life, and promote healthy behavior and health awareness. Automatic integration of information from m-Health systems into research databases can provide medical community possibility of data mining of huge amounts of data. This will allow improved insights into disease evolution, the rehabilitation process, and the effects of drug therapy.

\section{REFERENCES}

[1] R.S.H. Istepanian, E. Jovanov, Y.T. Zhang, "Guest Editorial Introduction to the Special Section on M-Health: Beyond Seamless Mobility and Global Wireless Health-Care Connectivity," IEEE Transactions on Information Technology in Biomedicine, Dec. 2004, 8(4): 405 - 414.

[2] D. Raskovic, T. Martin, E. Jovanov, "Medical Monitoring Applications for Wearable Computing," The Computer Journal, July 2004, 47(4): 495-504.

[3] National Alliance for Caregiving and American Association of Retired Persons, Family Caregiving in the United States, 1997.

[4] B.G. Steele, B. Belza, K. Cain, C. Warms, J. Coppersmith, J. Howard, "Bodies in motion: Monitoring daily activity and exercise with motion sensors in people with chronic pulmonary disease," Journal of Rehabilitation Research \& Development, Sep/Oct 2003, Supplement 2, 40(5): $45-58$.

[5] E. Jovanov, A. Milenković, C. Otto, P. De Groen, B. Johnson, S. Warren, and G. Taibi "A WBAN System for Ambulatory Monitoring of Physical Activity and Health Status: Applications and Challenges," in this Proceedings.

[6] S. Warren, "Beyond Telemedicine: Infrastructures for Intelligent Home Care Technology," Pre-ICADI Workshop on Technology for Aging, Disability, and Independence, The Royal Academy of Engineering, Westminster, London, England, June 2003.

[7] Bluetooth, http://www.bluetooth.org

[8] ZigBee, http://www.zigbee.org

[9] B.P. Otis, JM Rabaey, "A 300- $\mu \mathrm{W}$ 1.9-GHz CMOS Oscillator Utilizing Micromachined Resonators," IEEE Journal of Solid-State Circuits, July 2003, 38(7): 1271-1274. 